\title{
EXACT ANALYSIS FOR A CLASS OF SIMPLe, CIRCUIT-SWITCHED NETWORKS WITH BLOCKING
}

\author{
YAAKOV KOGAN, ${ }^{*}$ Technion-IIT
}

\begin{abstract}
We consider the same circuit switching problem as in Mitra [1]. The calculation of the blocking probabilities is reduced to finding the partition function for a closed exponential pseudo-network with $L-1$ customers. This pseudo-network differs from that in [1] in one respect only: service rates at nodes $1,2, \cdots, p$ depend on the queue length. The asymptotic expansion developed in [1] follows from our exact expression for the partition function.
\end{abstract}

\section{GENERATING AND PARTITION FUNCTIONS}

\section{Introduction}

Consider the same circuit switching, with blocking, as in Mitra's network [1]. There are $K_{j}$ lines from center $j$ to a hub, center $(p+1), 1 \leqq j \leqq p$ and $K_{p+1}$ lines from the hub to the destination, center $(p+2)$. A call originating at center $j$, of class $j$, requires two lines, one line from center $n$ to the hub, another line from the hub to the destination. The holding times for circuits of class $j$ are independent random variables with an arbitrary distribution and mean $1 / \mu_{j}$. At the termination of a call, both links are simultaneously released. The total offered traffic of call-requests at center $j, 1 \leqq j \leqq p$, is Poisson with rate parameter $\lambda_{j}$. A call-request at center $j$ may be blocked either if all lines from center $j$ to center $(p+1)$ are in use, or if all lines from center $(p+1)$ to center $(p+2)$ are in use. Blocked calls are cleared. The problem is the calculation of equilibrium blocking probabilities at each of the originating centers $1,2, \cdots, p$.

Let

and consider the case

$$
\rho_{j} \triangleq \lambda_{j} / \mu_{j}, 1 \leqq j \leqq p,
$$

$$
L \triangleq \sum_{j=1}^{p} K_{j}-K_{p+1} \leqq 1 .
$$

Let $n_{j}, 1 \leqq j \leqq p$, denote the number of calls of class $j$ in progress, and write $n=$ $\left(n_{1}, \cdots, n_{p}\right)$. Then the unique equilibrium distribution

where

$$
\pi(\boldsymbol{n})=\frac{1}{G} \prod_{j=1}^{p} \frac{\rho_{j}^{n_{j}}}{n_{j} !} \quad n \in \mathscr{S}
$$

$$
\mathscr{S}=\left\{0 \leqq n_{j} \leqq K_{j}, 1 \leqq j \leqq p, \text { and } \sum_{j=1}^{p} n_{j} \leqq K_{p+1}\right\} \text {, }
$$

and $G$ is the normalizing constant. $G$ is the partition function

$$
G(K ; L)=G\left(K_{1}, \cdots, K_{p} ; L\right) \triangleq \sum_{n \in \mathscr{S}} \prod_{j=1}^{p} \frac{\rho_{j}^{n_{j}}}{n_{j} !} .
$$

Received 21 February 1989.

* Postal address: Faculty of Industrial Engineering and Management, Technion-Israel Institute of Technology, Haifa 32 000, Israel.

Research supported by a grant from the Israeli Ministry of Communications. 
The equilibrium probability that a call of class $j$ is not blocked is

$$
\frac{G\left(K_{1}, \ldots, K_{j}-1, \ldots, K_{p} ; L\right)}{G\left(K_{1}, \ldots, K_{j}, \ldots, K_{p} ; L\right)}
$$

In [1] it is shown that

where

$$
G(K ; L)=\left[\prod_{j=1}^{p} \rho_{j}^{K_{j}} / K_{j} !\right]\left[\prod_{j=1}^{p} B^{-1}\left(K_{j}, \rho_{j}\right)-I(K ; L)\right]
$$

$$
B\left(K_{j}, \rho_{j}\right) \triangleq\left[\rho_{j}^{K_{j}} / K_{j} !\right] / \sum_{n=0}^{K_{j}}\left(\rho_{j}^{n} / n !\right)
$$

and a rather cumbersome procedure is given for generating the coefficients $A_{n}(L)$ of the complete expansion of $I(K ; L)$ in terms of the inverse powers of a parameter $N \gg 1$. The parameter $N$ is introduced in such a way that

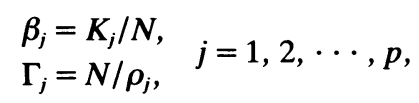

are quantities of order 1.

We show that for a fixed $K, I(K ; L)$ is the partition function for a closed exponential pseudo-network with a single class of $L-1$ customers. This pseudo-network differs from that in [1] in one respect only: service rate $v_{j}$, at node $j, 1 \leqq j \leqq p$, is dependent on the queue length $l_{j}$ and is given by

$$
v_{j}=v_{j}\left(l_{j}\right)=\frac{\rho_{j}}{K_{j}}\left(1-\frac{l_{j}-1}{K_{j}}\right)^{-1}=\Gamma_{j}^{-1}\left(\beta_{j}-\frac{l_{j}-1}{N}\right)^{-1} .
$$

Thus, the large parameter $N$ does not interfere with the calculation of the partition function of the pseudo-network. Moreover, the expansion of $I(K ; L)$ derived in [1] follows easily from the exact expression for this partition function, but our derivation is considerably shorter and simpler than Mitra's.

\section{Main result}

We start with the explicit expression for generating function

$$
\begin{aligned}
\mathscr{G}_{(\mathrm{z} ; \mathrm{x})} & \triangleq \sum_{L=0}^{\infty} \sum_{K_{1}=0}^{\infty} \cdots \sum_{K_{p}=0}^{\infty} x^{L} z_{1}^{K_{1}} \cdots z_{p}^{K_{1}} G(K ; L) \\
& =\frac{e^{\Sigma_{j} \rho_{j} z_{j}}}{1-x}\left[1 / \prod_{j=1}^{p}\left(1-z_{j}\right)-x / \prod_{j=1}^{p}\left(1-x z_{j}\right)\right]
\end{aligned}
$$

given in [1]. Denote by $\mathscr{J}(z ; x)$ the generating function of the partition function

where

$$
J(K ; L)=I(K ; L) \prod_{j=1}^{r}\left(\rho_{j}^{K_{j}} / K_{j} !\right)
$$

$$
I(K ; L)=\sum_{0 \leqq n \leqq K} 1_{l n \leqq L-1} \prod_{j=1}^{p} \frac{K_{j} !}{\left(K_{j}-n_{j}\right) !}\left(1 / \rho_{j}\right)^{n_{j}} .
$$

Then one can easily see that

$$
\mathscr{J}(z ; x)=\frac{x}{1-x} \prod_{j=1}^{p} \frac{e^{\rho_{j} z_{j}}}{1-x z_{j}}
$$


By Cauchy's formula

Denote

$$
J(K ; L)=(2 \pi i)^{-(p+1)} \oint_{C_{x}} \frac{x^{-L}}{1-x} d x \prod_{j=1}^{p} \oint_{C_{j}} \frac{z_{j}^{-K_{j}-1} e^{\rho_{j j} z_{j}}}{1-x z_{j}} d z_{j} .
$$

$$
R(x)=\prod_{j=1}^{p} \oint_{C_{j}} \frac{z_{j}^{-K_{j}-1} e^{\rho_{j} z_{j}}}{1-x z_{j}} d z_{j}
$$

Then

$$
J(K ; L)=\oint_{C_{x}} \frac{x^{-L} R(x)}{1-x} d x=\left.\frac{1}{(L-1) !} \frac{d^{L-1}\left[R(x)(1-x)^{-1}\right]}{d x^{L-1}}\right|_{x=0} .
$$

We now show that

$$
R(x)=\left(\prod_{j=1}^{p}\left(\rho_{j}^{K_{j}} / K_{j} !\right)\right) \prod_{j=1}^{p} f_{j}(x)
$$

where

Define the function

$$
f_{j}(x)=\rho_{j} \int_{0}^{\infty} e^{-\rho_{j} t}\left(1+x t_{j}\right)^{K_{j}} d t_{j}
$$

It is easily seen that

$$
g(x ; M) \triangleq \sum_{n_{1}+n_{2}=M} \frac{\rho^{n_{1}}}{n_{1} !} x^{n_{2}}
$$

$$
\sum_{M=0}^{\infty} z^{M}\left(g(x ; M)=e^{\rho z} /(1-x z)\right.
$$

and

$$
\begin{aligned}
g(x ; M) & =\frac{\rho^{M}}{M !} \sum_{n=0}^{M} \int_{0}^{\infty} e^{-t}\left(\begin{array}{c}
M \\
n
\end{array}\right)\left(\frac{t x}{\rho}\right)^{n} d t \\
& =\frac{\rho^{M}}{M !} \rho \int_{0}^{\infty} e^{-\rho t}(1+x t)^{M} d t .
\end{aligned}
$$

Now (3) is implied by (2) and (4)-(6). Thus we have

where

$$
I(K ; L)=\left.\frac{1}{(L-1) !} \frac{d^{L-1}\left[F(x)(1-x)^{-1}\right]}{d x^{L-1}}\right|_{x=0}
$$

$$
F(x)=\prod_{j=1}^{p} f_{j}(x)
$$

Leibnitz's rule for differentiating the product of several functions yields

where

$$
\begin{aligned}
I(K ; L) & =\frac{1}{(L-1) !} \sum_{m=0}^{L-1}(L-1) F^{(m)}(0)(L-1-m) ! \\
& =\sum_{m=0}^{L-1}\left(F^{(m)}(0) / m !\right)
\end{aligned}
$$

$$
F^{(m)}(0)=\sum_{l_{1}+\cdots l_{p}=m} \frac{m !}{l_{1} ! \cdots l_{p} !} \prod_{j=1}^{p} f_{j}^{\left(l_{j}\right)}(0)
$$

while

$$
f_{j}^{(l)}(0)=\frac{\left(K_{j}\right)_{l} l !}{\rho_{j}^{l}}=l ! \Gamma_{j}^{l} \prod_{i=0}^{l-1}\left(\beta_{j}-\frac{i}{N}\right)
$$


$\left(\right.$ Here $\left.\left(K_{j}\right)_{l}=K_{j}, \cdots,\left(K_{j}-l+1\right)\right)$. The final result is implied by (7)-(9) and given by

$$
I(K ; L)=\sum_{m=0}^{L-1} \sum_{l_{1}+\cdots+l_{p}=m} \prod_{j=1}^{p} \Gamma_{j}^{j_{j}} \prod_{i=1}^{l_{j}}\left(\beta_{j}-\frac{i-1}{N}\right) .
$$

It is easily seen that $(10)$ is the partition function for the closed exponential network with $p+1$ tandem queues and $L-1$ customers of a single class. The queueing discipline in each queue is FCFS. The service rate at queue $(p+1)$ is 1 while the service rate $v_{j}$ at queue $j, 1 \leqq j \leqq p$, is dependent on the queue length $l_{j}$ and given by $(1)$. Thus $I(K ; L)$ may be computed by well-known recursive formulas. Starting from (11), one can easily obtain the same final formulae for the expansion coefficients $A_{n}(L)$ as in [1].

\section{Reference}

[1] Mrtra, D. (1987) Asymptotic analysis and computational methods for a class of simple, circuit-switched networks with blocking. Adv. Appl. Prob. 19, 219-239. 